\title{
Correspondence
}

\section{Successful transplantation of CD34 + selected peripheral blood stem cells from an unrelated donor in an adult patient with Diamond- Blackfan anemia and secondary hemochromatosis}

\author{
Bone Marrow Transplantation (2005) 35, 99-100.
} doi:10.1038/sj.bmt.1704700

Published online 1 November 2004

Diamond-Blackfan anemia (DBA) is a rare congenital or early-onset pure red cell aplasia. Approximately $60-70 \%$ of patients with DBA respond initially to corticosteroid therapy. Many patients require high-dose steroids long term or develop resistance to therapy and require transfusions long term. Hemopoietic stem cell transplantation (HSCT) has been used as a potentially curative therapy in patients with DBA. Results of allogeneic matched sibling HSCT are encouraging, but poor outcomes are reported in patients receiving alternative donor stem cell transplants because of high incidence of transplant-related mortality (for a review, see Vlachos et $a l^{1}$ ). Therefore, new transplantation modalities with lower risk of GVHD and lower organ toxicity have to be studied, especially in patients who lack HLA-identical siblings. We report the first case of a successful unrelated CD34 + selected PBSCT in a steroid-resistant adult with DBA and severe iron overload after a dose-reduced conditioning regimen.

DBA had been diagnosed in our patient at 2 months of age. The disease initially responded to steroids. Transfusion dependence occurred at the age of 15. Allogeneic PBSCT from an unrelated donor was planned after 7 years on a chronic transfusion-chelation program. At transplantation, the patient presented without physical abnormalities but short stature (height $1.52 \mathrm{~m}$, weight $42 \mathrm{~kg}$ at the age of 22). Leukocytes and platelets were normal and red cell transfusions were required every 3 weeks. Ferritin was $>7000 \mu \mathrm{g} / 1$ and marked iron deposition in the liver could be shown by MRI associated with elevated liver enzymes. Echocardiography revealed myocardiopathy-associated heart failure (assessed ejection fraction 30-35\%).

The conditioning regimen comprised fludarabine $30 \mathrm{mg} / \mathrm{m}^{2}$ once daily for 4 consecutive days, 8 Gy TBI (4 fractions of $2 \mathrm{~Gy}$ twice daily on 2 consecutive days) and antithymocyte globulin (ATG Fresenius $10 \mathrm{mg} / \mathrm{kg}$ i.v. daily from day -5 to -2 ). The donor was allele-matched for HLA-A, -B, -C, -DRB1 and -DQB1. After immunomagnetic CD34 + selection of G-CSF mobilized stem cells, the graft contained $6.43 \times 10^{6} \mathrm{CD} 34+$ cells $/ \mathrm{kg}$ and $<1 \times 10^{4}$ $\mathrm{CD} 3+$ cells $/ \mathrm{kg}$. Cyclosporine was administered as prophylaxis of GvHD and graft rejection. No regimen-related toxicity was seen, and engraftment occurred on day 17. Complete donor chimerism was demonstrated ${ }^{2}$ from day 14 to the last visit at 28 months. No acute or chronic GVHD, or opportunistic infections have been seen. Hemoglobin values have been normal since day 49 post transplant

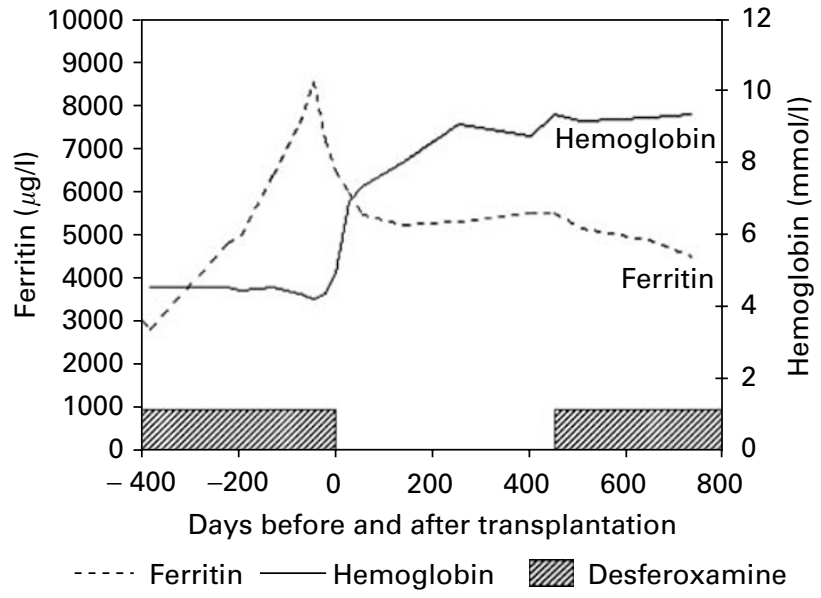

Figure 1 Levels of hemoglobin (right $y$-axis, solid line) and serum ferritin (left $y$-axis, dotted line) pre and post HSCT and its dependence on the desferoxamine chelation program (scratched area) PDF ( $7 \mathrm{~kb})$.

(Figure 1); the last transfusion was 11 days after HSCT. Deferoxamine chelation was continued after HSCT, resulting in a decline in serum ferritin to $4521 \mu \mathrm{g} / 126$ months after HSCT (Figure 1). Heart function improved significantly with normalization of the echocardiogram.

HSCT has been restricted in heavily transfused patients because of allosensitization and expectation of substantial treatment-related toxicity due to iron overload leading to heart and liver damage. To reduce the risk of severe organ toxicity and GvHD, we decided to use a dose-reduced conditioning regimen and a CD $34+$-selected graft. The high dose of $\mathrm{CD} 34+$ cells and the low number of contaminating $\mathrm{T}$ cells contained in the positive fraction allow for stable engraftment of PBPC from unrelated and partially mismatched donors without an increased rate of acute and chronic GvHD. ${ }^{3,4}$ Our patient experienced a decrease of serum ferritin and a significant improvement of heart function with continuation of deferroxamine chelation after HSCT. This suggests that, although the optimum time for transplantation is after a patient becomes refractory to corticosteroids, extensive transfusion is not necessarily a contraindication to allogeneic HSCT.
A Berndt
Medizinische Klinik und
A Helwig
G Ehninger
Poliklinik I, Universitätsklinikum
M Bornhäuser
Carl Gustav Carus, Fetscherstr 74
01307 Dresden, Germany

\section{References}

1 Vlachos A, Federman N, Reyes-Haley C et al. Hematopoietic stem cell transplantation for Diamond Blackfan anemia: a report from the Diamond Blackfan Anemia Registry. Bone Marrow Transplant 2001; 27: 381-386. 
2 Thiede C, Florek M, Bornhauser M et al. Rapid quantification of mixed chimerism using multiplex amplification of short tandem repeat markers and fluorescence detection. Bone Marrow Transplant 1999; 23: 1055-1060.

3 Aversa F, Tabilio A, Velardi A. Treatment of high-risk acute leukemia with T-cell-depleted stem cells from related donors with one fully mismatched HLA haplotype. N Engl J Med 1998; 339: 1186-1193.

4 Lang P, Handgretinger R, Niethammer D et al. Transplantation of highly purified CD34+ progenitor cells from unrelated donors in pediatric leukemia. Blood 2003; 101: $1630-1636$ 